\title{
polo, a mitotic mutant of Drosophila displaying abnormal spindle poles
}

\author{
CLAUDIO E. SUNKEL and DAVID M. GLOVER*
}

Cancer Research Campaign, Eukaryotic Molecular Genetics Research Group, Department of Biochemistry, Imperial College of Science and Technology, London SW7 2AZ, LiK

* Author for correspondence

\section{Summary}

Neuroblast cells in larvae homozygous for mutant alleles of the locus polo show a high frequency of metaphases in which the chromosomes have a circular arrangement, and anaphase fig. ures in which chromosomes appear to be randomly oriented with respect to at least one of the spindle poles. These defects appear to lead to the production of polyploid cells. Sex chromosome disjunction is affected in male meiosis, primarily in the second division, and the meiotic spindles of living cells are abnormal. One allele is a larval lethal, whereas another is semi-lethal with about $7 \%$ of homozygotes surviving as adults. Embryos from homozygous polo females have aberrant mitotic spindles that are highly branched and have broad poles. Immunofluorescence studies with an antibody that recognizes an antigen associated with the centrosome indicate that the organization of this organelle is disrupted in the mutant embryos.

Key words: Drosophila, mitotic mutant, spindle poles.

\section{Introduction}

Genetic approaches have been highly successful in analysing the cell cycle in yeasts, leading to a detailed pathway of the events of mitosis (see, e.g., Hartwell et al. 1974; Pringle \& Hartwell, 1981; Beach et al. 1982; Nurse, 1985). However, aspects of the mechanics of mitosis differ in yeasts from those in the multicellular eukaryotes, and furthermore there are additional constraints upon cell division in higher organisms because of the need to maintain temporal and spatial control of cell proliferation.

Drosophila offers considerable advantages as an organism in which to study cell division. Work by Baker and his colleagues has shown that certain loci originally identified by meiotic mutants, and mutants showing increased mutagen sensitivity, encode products that function in mitosis (Baker et al. 1978; Baker \& Smith, 1979). Additional mitotic mutants have been identified by their effects on chromosome integrity and fidelity of mitotic chromosome transmission (Baker et al. 1982; Smith et al. 1985). There are two major stages of Drosophila development at which one can expect to detect mutations affecting mitotic cell division. The first is during early embryogenesis, when there are 13

Journal of Cell Science 89, 25-38 (1988)

Printed in Great Britain (C) The Company of Biologists Limited 1988 nuclear divisions at about 10 -min intervals before individual nuclei undergo cellularization (Zalokar \& Erk, 1976). During this time there is little or no zygotic gene expression, and so one might therefore expect a class of maternal effect lethal mutations that disrupt the functions of the genes encoding these components. There are then only three to four more cell divisions during embryogenesis and most of larval development involves cell growth, with the endoreduplication of DNA in the absence of mitosis. Imaginal cells, destined to form the adult organism and not themselves necessary for the survival of the larva, divide throughout larval development, as do cells of the nervous system. A second class of mitotic mutations can therefore be recognized in which the imaginal cells do not proliferate within the larva and death ensues during the late larval or early pupal stages. This late larval lethal phenotype of mutations in essential mitotic functions was first recognized in the analysis of repair-defective mutants (Baker et al. 1982).

We have begun to screen the embryos of flies having maternal effect mutations in order to identify aberrant mitoses during early embryogenesis. We expect that such mutations might fall into distinct categories, corresponding to: genes expressed only during 
oogenesis to provide a product specific for the cleavage embryo; and genes that are expressed not only at oogenesis but are also required for diploid cell proliferation in the imaginal and neuroblast cells of the larvae. The mutation gnu appears to be an example of the former category. This mutation results in uncontrolled DNA replication, leading to the formation of giant nuclei, whilst the division cycle of the centrosomes appears not to be affected (Freeman et al. 1986). This phenotype also develops in unfertilized eggs, indicating that the gene plays a role in the correct establishment of zygotic development (Freeman \& Glover, 1987). In this paper we describe a mutation, polo', the wild-type function of which is required both in the early embryo and in the diploid cells of the larva. Using immunofluorescence techniques we find that the mitotic spindles in polo embryos (from homozygous polo ${ }^{1}$ females) are highly branched and have defective poles. Homozygous larvae also exhibit aberrant mitotic divisions in their brain cells and in addition we have observed abnormal meiosis in homozygous males. A stronger allele, polo ${ }^{2}$, causes the death of homozygous larvae.

\section{Materials and methods}

\section{Genetic variants}

The mutation polo ${ }^{1}$ was isolated by Nusslein-Volhard in a genetic screen designed to select sterile females. The chromosome used was ru st e ca mutagenized with ethylmethylsulphonate (EMS). polo ${ }^{\prime}$ is a recessive mutation that maps to the left arm of chromosome 3 near the centromere at 3-46 on the basis of 112 recombinants between Lyra (3-40.5) and Stubble (58.2). The original polo ${ }^{1}$ mutation is recessive and some $7 \%$ of expected homozygotes survive to eclose.

The allele, polo ${ }^{2}$, was found among a collection of $\mathrm{P}$ element-induced lethals isolated by R. Karess. All other stocks were decribed by Lindsley \& Grell (1968). All stocks were grown at $25^{\circ} \mathrm{C}$ under standard culture conditions and media.

\section{Neuroblast preparations}

Cytological preparations were made from late third-instar larvae that had been grown at $25^{\circ} \mathrm{C}$, collected and then washed in saline. They were dissected in $0.7 \% \mathrm{NaCl}$ and the brain was transferred to a drop of $45 \%$ acetic acid for $15 \mathrm{~s}$. The tissue was then placed for a further $15 \mathrm{~s}$ in $3 \%$ orcein dissolved in $45 \%$ acetic acid and then cleared of excess stain in $60 \%$ acetic acid. The brain was then placed in a small drop of $3 \%$ orcein in $60 \%$ acetic acid and squashed firmly. Preparations were observed under phase-contrast optics. Quantification of mitotic figures was carried out essentially as described by Gonzalez (1986). The unit used was the area of a brain-squash that can be seen under the microscope with a $63 \times$ Zeiss objective and $10 \times$ eyepieces. The number of metaphases and the number of anaphases per field was determined for at least 100 fields from 15 brains for polo 1 polo ${ }^{1}$ or polo ${ }^{1} /+$.
Preparations were also made by incubating dissected brains in $0.4 \%$ colchicine in $0.7 \% \mathrm{NaCl}$ for $1 \mathrm{~h}$ before staining. After washing in saline the brains were incubated for $15 \mathrm{~min}$ in $30 \mathrm{~mm}$-sodium citrate and then stained and squashed as before.

\section{Live testis squashes}

The pharate adults or recently emerged adult males were dissected in $0.7 \% \mathrm{NaCl}$ on a siliconized slide. The whole testis was placed in a large drop of $0.7 \% \mathrm{NaCl}$ and cut in half across the middle using dissecting needles. A small nonsiliconized coverslip was placed on top and the whole preparation observed under phase-contrast with a $20 \times$ objective. The squash is performed by withdrawing excess liquid with blotting paper by capillarity. Live squashes were photographed within 10-20 min. Measurement of nuclear diameters for controls or polo ${ }^{1}$ homozygotes was done by measuring at least 100 nuclear diameters from photographic prints of 10 different preparations.

\section{Fixation and staining of embryos}

Embryos were fixed and stained exactly as described by Freeman et al. (1986). Taxol was used to stabilize microtubules exactly as described in that paper and by Karr \& Alberts (1986). We are well aware of potential artefacts that can arise from the use of taxol and were discussed at length in these two papers. In our hands the staining of wild-type embryos under these conditions gives a pattern of tubulin staining in agreement with the results of Karr \& Alberts (1986).

\section{Antibodies}

The antibodies used in this study were the following: anticentrosome antibody, Bx63 (Frash et al. 1987); anti-tubulin antibody, YL1/2 (Kilmartin et al. 1982); and anti-lamin antibody, T47 (Dequin et al. 1984). Rhodamine- and fluorescein-conjugated second antibodies were bought from Jackson Immunoresearch Laboratories Inc. USA

\section{Results}

\section{The somatic phenotype}

We describe the phenotypes of two mutant alleles of a locus, polo, which maps close to the centromere at 46 on the third chromosome of Drosophila melanogaster. About $7 \%$ of flies homozygous for the original, EMS. induced allele eclose, and the eggs laid by homozygous females arrest early in development. We selected the mutation for further study since these embryos showed an abnormal distribution of nuclei, leading us to suspect a mitotic abnormality. The second, more extreme, allele $\left(p o l o^{2}\right)$ is a $\mathrm{P}$-element-induced recessive larval lethal, which is, however, viable as a transheterozygote with polol.

We have studied the effect of polo' or polo ${ }^{2}$ on chromosome integrity and segregation in the diploid neuroblasts of third-instar larvae. Our analysis of these cytological preparations shows that the frequency of 
metaphases per field is lower in homozygous polo ${ }^{1}$ larvae than in wild-type larvae (see Materials and methods and legend to Fig. 1). The frequency of anaphases per field is also lower, but the relative proportion of metaphases to anaphases is roughly the same. About $70 \%$ of the metaphase figures show abnormalities (Fig. 1), which can be classified into three classes. Two classes show circular arrangements of the chromosomes. In one case there is a normal chromosomal complement (Fig. 1A). Homozygous polo larvae have about half of their mitotic figures in this configuration, compared to approximately $4 \%$ of the mitotic figures in wild-type larvae. In the second class, the nucleus is polyploid (Fig. 1B,C). Such polyploid figures are never seen in wild-type brains. In both circular configurations, the dot-like fourth chromosomes are seen in the centre of the mitotic figure with the major autosomes and sex chromosomes being arranged around them. We are not able to say categorically whether these figures are in metaphase or if they have proceeded to anaphase. We have, however, counted them as metaphases in our numerical analyses (see legend to Fig. 1). We do not observe circular mitotic figures, polyploid or otherwise, when the squashes are made in the presence of colchicine, indicating the requirement for functional microtubules for their formation (data not shown). The third class, which is much less frequent, shows aneuploid figures. Fig. 1D shows a cell that contains two extra chromosomes, a $Y$ and a fourth. We have seen no other chromosomal defects; chromosomes are never fragmented and they appear to undergo normal condensation.

About $90 \%$ of anaphase figures seen in polo $1 /$ polo $^{1}$ larval brains are clearly abnormal. In a typical example, shown in Fig. 1E, the chromosomes attached to one of the spindle poles appear to be randomly oriented and are not all lying with their telomeres pointing towards the middle of the spindle. This arrangement is sometimes observed in wild-type larvae but at a very low frequency. The majority of wild-type anaphases resemble the one shown in Fig. 1F, in which the chromosome arms are all oriented towards the opposite spindle pole. Occasionally we have been able to observe anaphases that are trying to separate polyploid nuclei.

These cytological abnormalities are reflected in the viability of homozygous polo' adults. The progeny that eclose do so on average 1.5 days later than their heterozygous siblings. The majority die either as late third instars or during pupariation. The polo ${ }^{2}$ allele shows a more extreme phenotype. None of the expected polo ${ }^{2}$ homozygotes survives, but they die as larvae during the early stages of the third instar. The cytological phenotypes of their larval neuroblasts are similar to polo' homozygotes. The heterozygotes polo $1 /$ polo $^{2}$ do eclose, but with a slightly lower frequency compared to polo' homozygotes. However, these trans-heterozygotes show very abnormal cuticle formation of the abdomen affecting both tergites and sternites.

\section{The meiotic phenotype}

We were interested to know whether or not the mutations also affected meiotic divisions and we chose to analyse this process in males homozygous for the original allele, which in contrast to males homozygous for polo ${ }^{2}$ are fertile. Not only the chromosomes, but also mitochondria, segregate to daughter nuclei upon the meiotic spindle in male germ cells. This association of the mitochondria with the meiotic spindle makes it possible to observe the spindle in living cells using phase-contrast optics. Meiotic spindles from wild-type and polo $1 /$ polo ${ }^{1}$ males are shown in Fig. 2. The wildtype cells undergoing meiotic division have very characteristic spindles with two clearly defined poles (Fig. 2A). However, the meiotic spindles found in polo $1 /$ polo ${ }^{1}$ testes are generally more irregular in shape and structure. These range from almost normal to tetrapolar spindles (Fig. 2B) or even multipolar ones (Fig. 2C).

A consequence of these abnormal spindle structures would be irregular chromosome distribution amongst the resulting gametes. Perhaps the easiest stage at which abnormal chromosomal segregation can be analysed is within the cyst of spermatids referred to as the 'onion stage'. Any differences in the DNA content of the post-meiotic products found at the onion stage would be reflected by the nuclear diameters of these cells (Ripoll et al. 1985; Gonzalez, personal communication). In wild-type spermatids two round organelles are clearly visible at this stage (Fig. 3A). The clear organelle is the post-meiotic nucleus, the diameter of which is remarkably constant amongst different gametes, reflecting their equal DNA content (Fig. 3B). Our measurements of the diameters of these nuclei are in good agreement with those of Gonzalez et al. (1987) and reflect a haploid DNA content. The dark organelle, the Nebenkern, consists of mitochondria that have been distributed between daughter cells on the meiotic spindle, and which are associated with tubulin. This complex will later elongate to form the sperm tail. Before elongation starts this organelle is also very regular in size and shape in the wild type (Fig. $3 A$ ). In the post-meiotic products of polo $1 /$ polol testes, most nuclei and associated Nebenkern appear abnormal (Fig. 3C). The histogram of nuclear diameters (Fig. 3D) indicates that there is a wide range of nuclear sizes. Gonzalez et al. (1987) have demonstrated that the nuclear diameter is a function of the DNA content. Comparison of the histogram in Fig. 3D with their standardized data indicates that the polo 
spermatids can contain from less than a quarter of the haploid DNA complement up to as much as a tetraploid complement. Furthermore, whilst in wild-type preparations there is always a single nucleus associated with one Nebenkern, there is no such correspondence in the mutant. The irregular size of the Nebenkern in polo ${ }^{1}$ homozygotes indicates unequal partitioning of
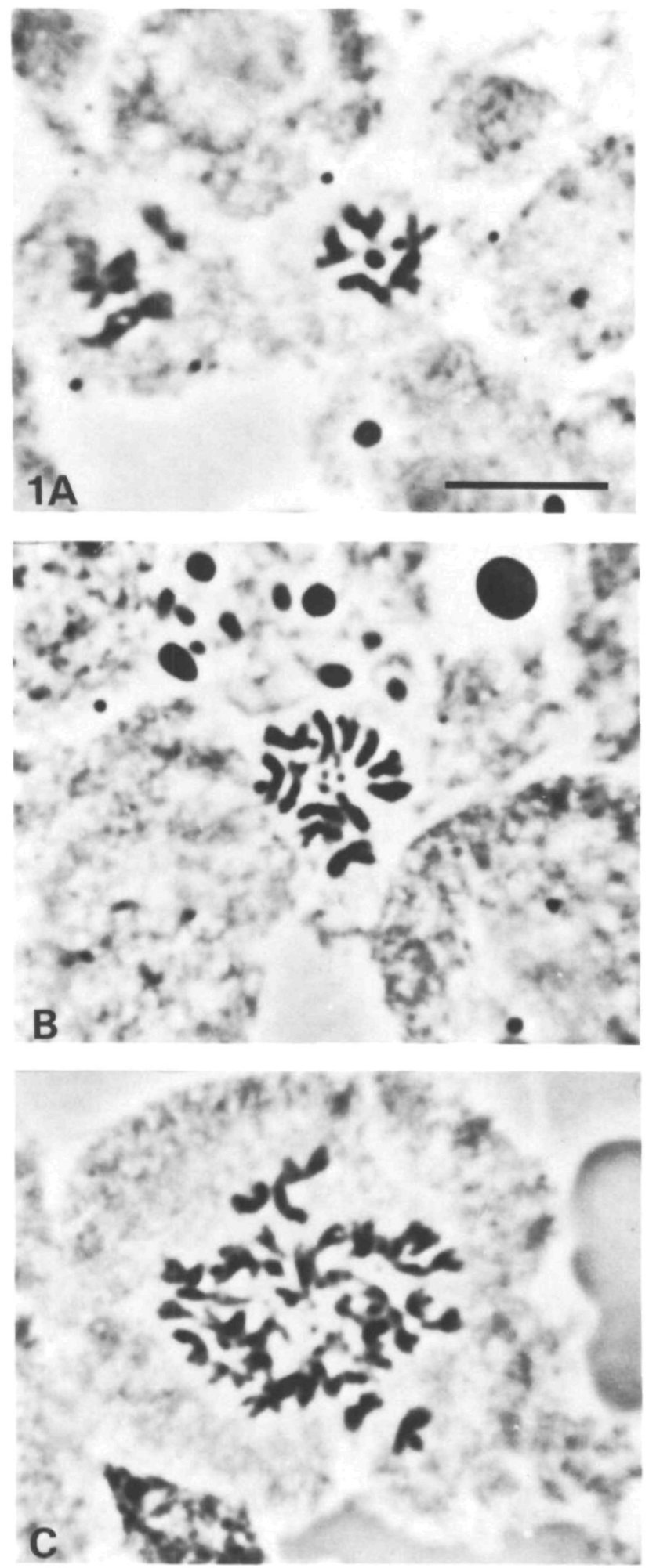

mitochondria into daughter cells on the meiotic spindles.

We have analysed male meiosis further by testing homozygote polo ${ }^{1}$ males for genetic non-disjunction of the sex chromosomes. This was carried out by crossing females carrying a $Y$ chromosome and two $X$ chromosomes sharing the same centromere $(C(1) y f / Y$ and also
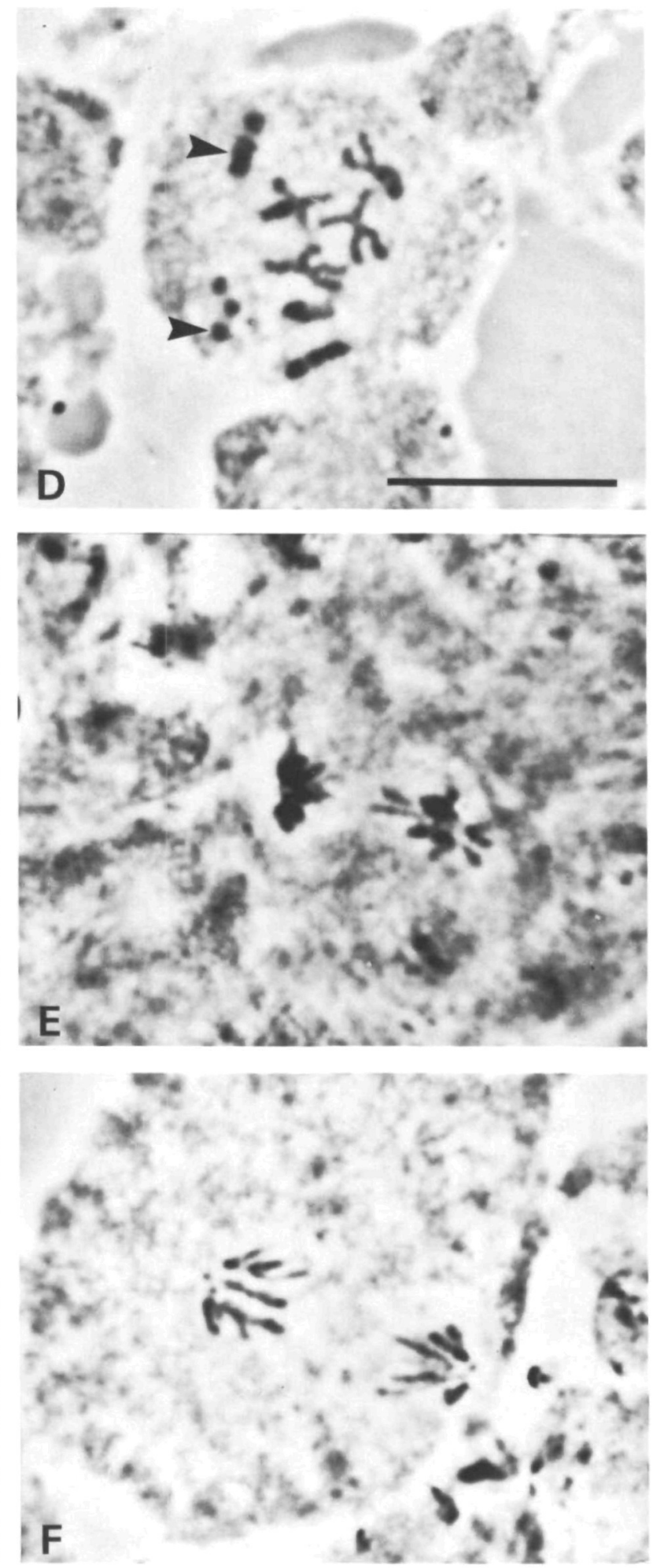
referred to as $\hat{\mathrm{XX}}, y f / Y)$ to males with marked $X$ and $Y$ chromosomes $\left(X w / Y B^{S}\right)$. Meiosis comprises two divisions, during the first of which homologous chromosomes are segregated to the spindle poles, the sister chromatids not being split. In the second division, the sister chromatids are separated and the gametes receive a haploid chromosome complement. If therefore disjunction occurs correctly, the viable progeny expected from this cross would be $w$ males in which the $X$ chromosome is derived from the father and the $Y$ from the mother $(X w / Y)$, and $y f B^{S}$ females, which have the attached $X$ of the mother and the marked $Y$ from the father $\left(\hat{\mathrm{X}} y \mathrm{f} / \mathrm{YB}^{\mathrm{s}}\right)$. Non-disjunction in the male parent during the first division results in gametes carrying both $\mathrm{X} w$ and $Y B^{S}$ chromosomes (see the diagram of Table 1). Gametes carrying no sex chromosomes can result from non-disjunction in the first or second divisions. The other products of non-disjunction in the second division are gametes carrying two free $\mathrm{X} z \mathrm{u}$ chromosomes or two free $Y B^{S}$ chromosomes (Table 1). Thus non-disjunction in the first meiotic division can be detected by the presence of $w B^{S}$ males $\left(X w / Y B^{s} / Y\right)$, and in the second division by $w$ females $(\mathrm{Xw} / \mathrm{Xw} / \mathrm{Y})$, whereas $y f$ females $(\hat{\mathrm{X} y} \mathrm{f} / \mathrm{O})$ are indicative of non-disjunction at either division.

In heterozygous polo $1 /+$ males non-disjunction was observed at a frequency of about $0.07 \%$, a level comparable to that in wild type (Sandler et al. 1968). Males homozygous for polo ${ }^{1}$ produce two major classes of abnormal diplo-gametes (Table 1). The predominance of $\mathrm{XX}$ gametes $(1.7 \%)$ relative to $\mathrm{XY}$ gametes $(0.23 \%)$ suggests that most of the aberrant segregation is occurring in the second meiotic division. The low

Fig. 1. Mitotic figures in brain neuroblasts of homozygous polo' larvae. We scored 871 metaphase figures (using the criteria described in Results) from the neuroblasts of polo 1 polo' larvae of which $25.6 \%$ appeared 'normal'. This contrasts with the proportion of normal figures in wild-type brains for which we scored $96.2 \%$ in a sample of 1249 . Homozygous polo' larvae had $47.6 \%$ of the metaphase figures in circular configurations of the type shown in $\mathrm{A}$. A significantly smaller proportion of wild-type cells (3.8\%) showed similar figures. Circular polyploid figures $(B, C)$ and aneuploid figures (D) were found in homozygous polo animals at frequencies of $20.1 \%$ and $6.9 \%$, respectively, and not at all in wild types. The arrowheads in D mark additional $Y$ and fourth chromosomes in this aneuploid cell. Of 230 anaphase figures in homozygous polo larvae, $14 \%$ were normal (F) compared with $89.6 \%$ in the 308 wild-type anaphases scored. 'Abnormal' anaphases, like the one shown in $\mathrm{E}$, were scored at a frequency of $86 \%$ in the mutant and $10.4 \%$ in wild-type neuroblast squashes. Note the random orientations of the chromosomes at the upper pole of the anaphase in $\mathrm{E}$. Bar in $\mathrm{A}, 5 \mu \mathrm{m}(\mathrm{A}, \mathrm{B}, \mathrm{C})$; Bar in $D, 5 \mu \mathrm{m}(\mathrm{D}, \mathrm{E}, \mathrm{F})$. These are aceto-orcein-stained squashes prepared and photographed as described in Materials and methods. frequency of nullo $(O)$ gametes within this sample is striking and contrasts with observations that have been made with most other mutants that affect meiosis in Drosophila (see review by Baker \& Hall, 1976; and the Discussion). It could be explained by the selective loss of these gametes. An alternative explanation would be that directed non-disjunction is occurring, so that, in addition to having no sex chromosomes, these sperm are also either missing autosomes or have additional autosomes and so would produce inviable zygotes.

In order to test this latter idea, we have looked for the simultaneous non-disjunction of the sex chromosomes and the second chromosome by crossing $X z / Y B^{S}$ males to females having wild-type $X$ chromosomes and a marked pair of second chromosomes attached through a common centromere (C(2)FN). These females will give rise to either nullo-2 or attached diplo-2 gametes and so progeny will survive only if non-disjunction has occurred in the male in order that the eggs are fertilized by diplo- 2 or nullo- 2 sperm, respectively. As the second chromosomes of the male are unmarked, we cannot distinguish between non-disjunction in the first or second meiotic divisions. The cross does, however, allow one to determine whether or not non-disjunction of the second chromosome is occurring and, if so, whether or not it is independent of non-disjunction of the sex chromosomes. Simultaneous non-disjunction of the sex chromosomes in the males should give the same set of gametes as described above, and would be recognizable by $X / O$ male progeny (from nullo-gametes produced in either the first or second meiotic divisions); and $\mathrm{X} / \mathrm{Xw} / \mathrm{YB}^{\mathrm{S}}$ females (indicative of $\mathrm{NY}$ gametes arising in the first meiotic division). $\mathrm{Nw} / \mathrm{Xw}$ gametes from the male would give triplo- $\lambda$ zygotes, which are not considered because of their poor and irreproducible viability.

As expected, non-disjunction does not occur in heterozygous polo/ + males, which consequently give no progeny when mated to $C(2) E N$ females (Table 2). The results with homozygous polo $/$ polo $^{1}$ males indicate that non-disjunction of the second chromosome does occur (Table 2). In those cases in which second chromosome non-disjunction is independent of the sex chomosomes (to give gametes having only one sex chromosome and being either diplo- 2 or nullo-2), one sees a higher recovery of diplo-2 gametes at the expense of nullo-2 gametes, indicating either their selective loss or their production at a lower frequency. Simultaneous non-disjunction of the second chromosome and the sex chromosomes occurs at a high frequency and in this cross is detected predominantly by nullo- 1 gametes. In those cases exhibiting simultaneous non-disjunction of both the second and sex chromosomes, we recovered only one $X w / Y B^{. \prime}$ gamete $(0.3 \%$ of total gametes recovered) from the males, indicating as with the first 

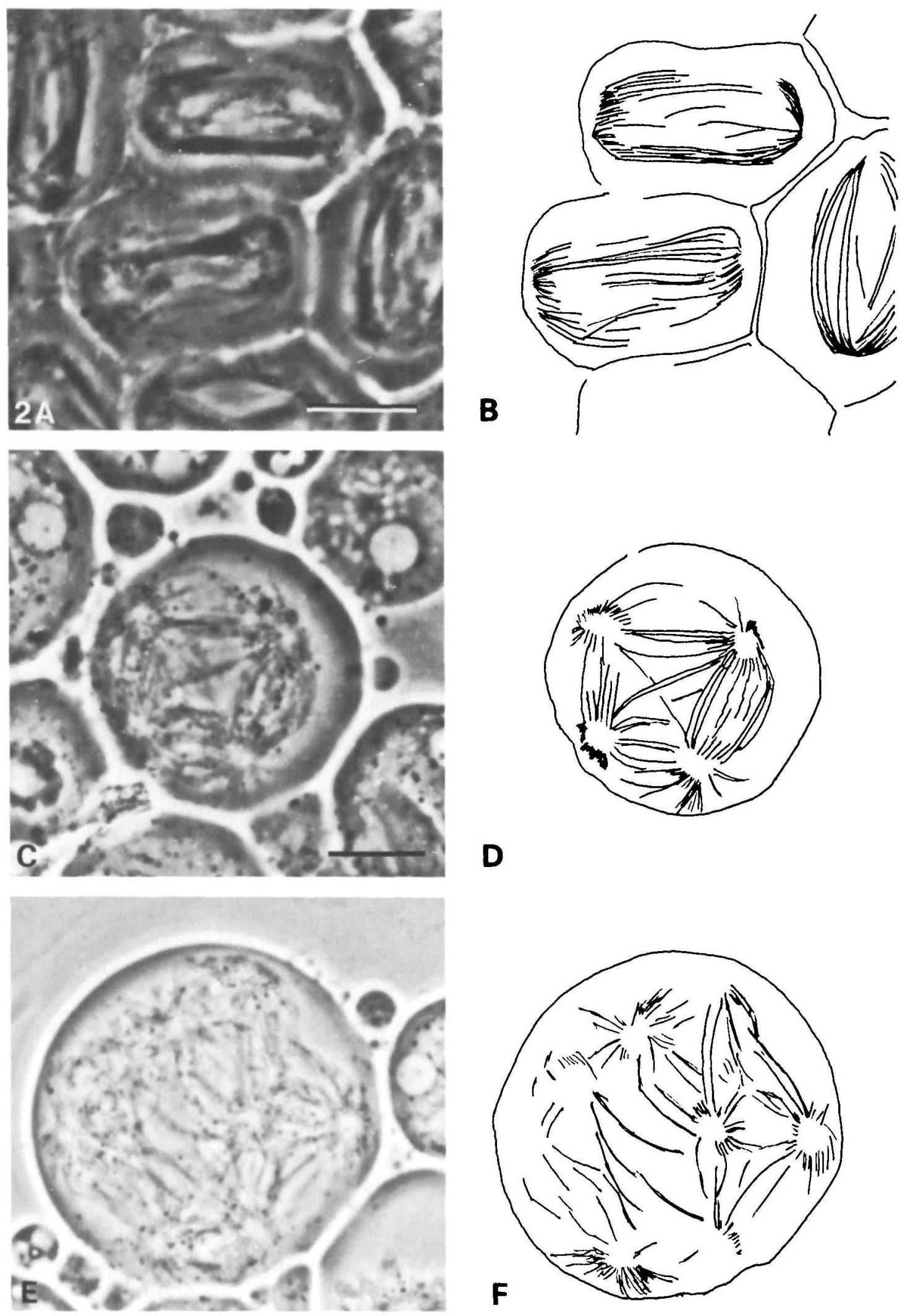

D

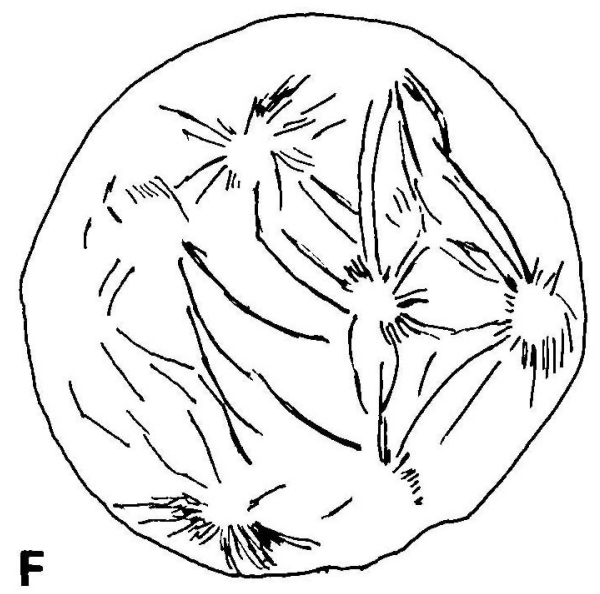

Fig. 2. Meiotic spindles in live cells during spermiogenesis. A,B. A cyst of wild-type cells undergoing the second meiotic division. C,D. A tetrapolar spindle in a homozygous polo ${ }^{1}$ cell undergoing meiosis. E,F. A multipolar meiotic spindle in a homozygous polo' cell. Details of the preparation are given in Materials and methods. Bars; A, $10 \mu \mathrm{m} ; \mathrm{C}, \mathrm{E}, 10 \mu \mathrm{m}$. 

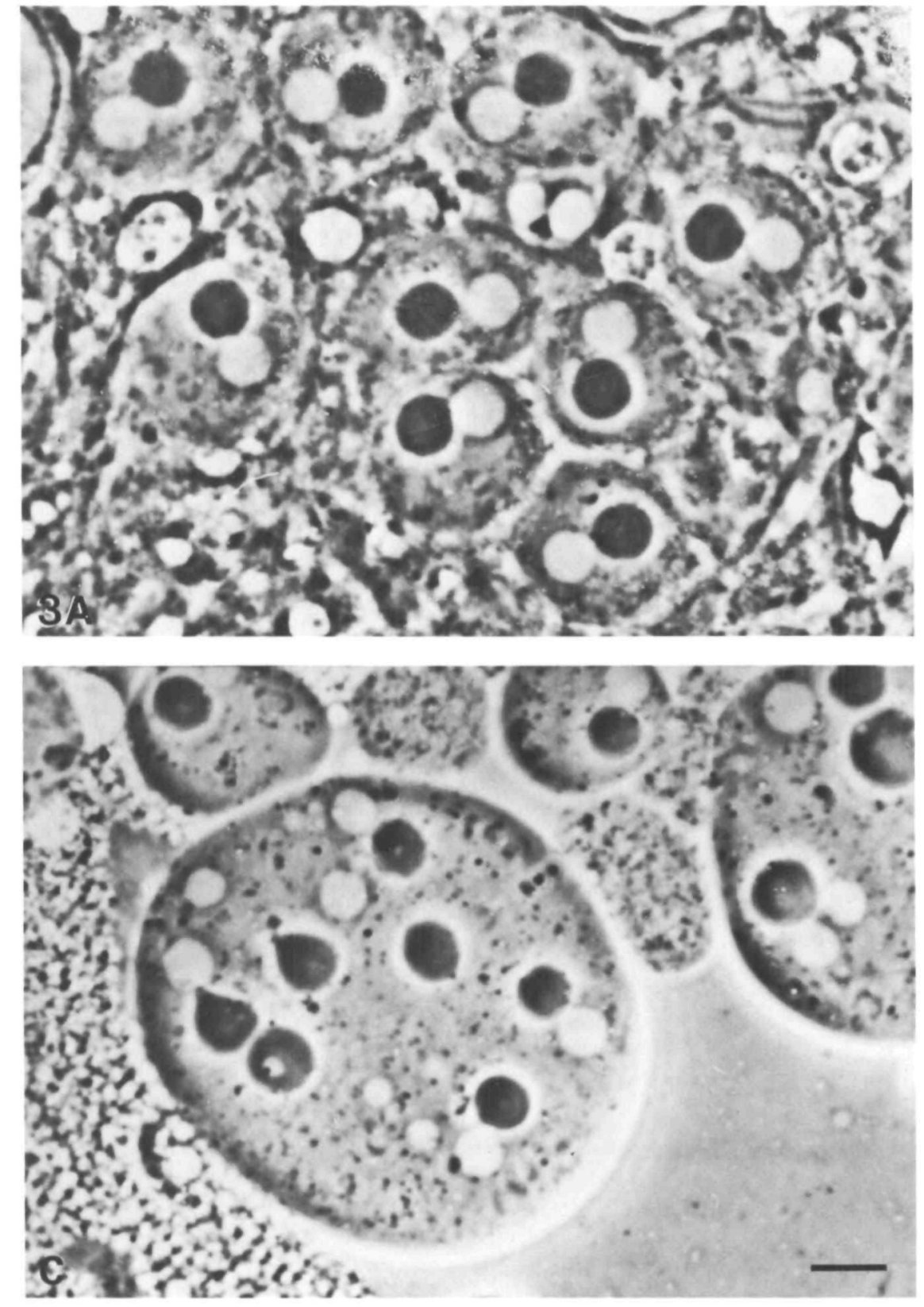

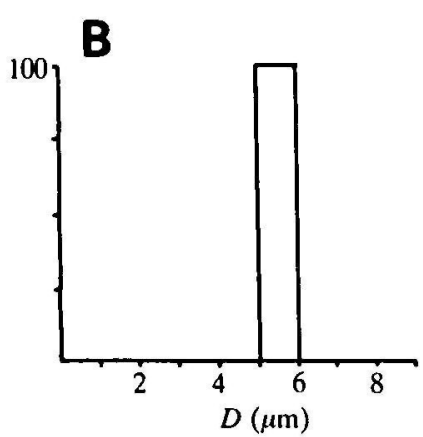

D

1001

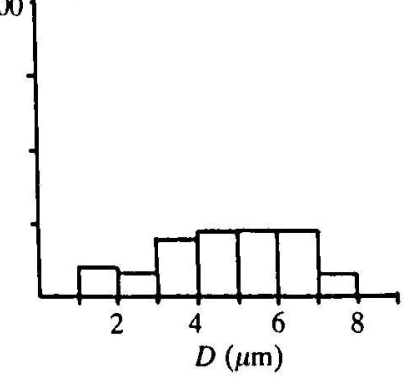

Fig. 3. Spermatids from polo' males. Spermatids from heterozygous $(A)$ or homozygous $(C)$ animals at the 'onion stage'. The Nebenkern appear as black circles, the nuclei as white circles. The histograms display the nuclear diameters $(D)$ from the heterozygous (B) and homozygous (D) animals. Bar, $5 \mu \mathrm{m}$. The occurrence of the cytological abnormalities depicted in both this and Fig. 2 correlates well with the decrease in fertility of polo $1 /$ polo males with age. Dissection of virgin males either at eclosion, or when they were 5 or 10 days old, revealed that the proportion bearing degenerated gonads increases from $18 \%$ to $23 \%$ and up to $50 \%$ after these respective intervals. polo $1 /$ polo $^{2}$ males show the same cytological phenotypes described for polo ${ }^{1}$ homozygotes. They were, however, unable to fertilize virgin Oregon $\mathrm{R}$ females. This could be a direct consequence of aberrant meiosis leading to the production of abnormal sperm, but it might also be a result of the cuticular abnormalities that affect the genitalia, since some sperm are found that have a normal appearance.

cross that the frequency of sex chromosome nondisjunction in the first meiotic division is low. Of the nullo- $X$ gametes indicating simultaneous non-disjunction, $95 \%$ are diplo- 2 rather than nullo-2. These data could be explained if the defect in polo males directed the preferential (but not absolute) disjunction of non-homologous chromosomes. In such a case, nullo- $X$ gametes would contain an extra complement of one of the autosomes, and so would not be detected in the first cross (Table 1), whereas the second cross allows one specifically to recover gametes that are nullo- $X$ and diplo-2. The dearth of nullo- $X ;$ nullo-2 
Table 1. Genetic non-disjunction test for the sex chromosomes in polo $1 /$ polo $^{1}$ and control males

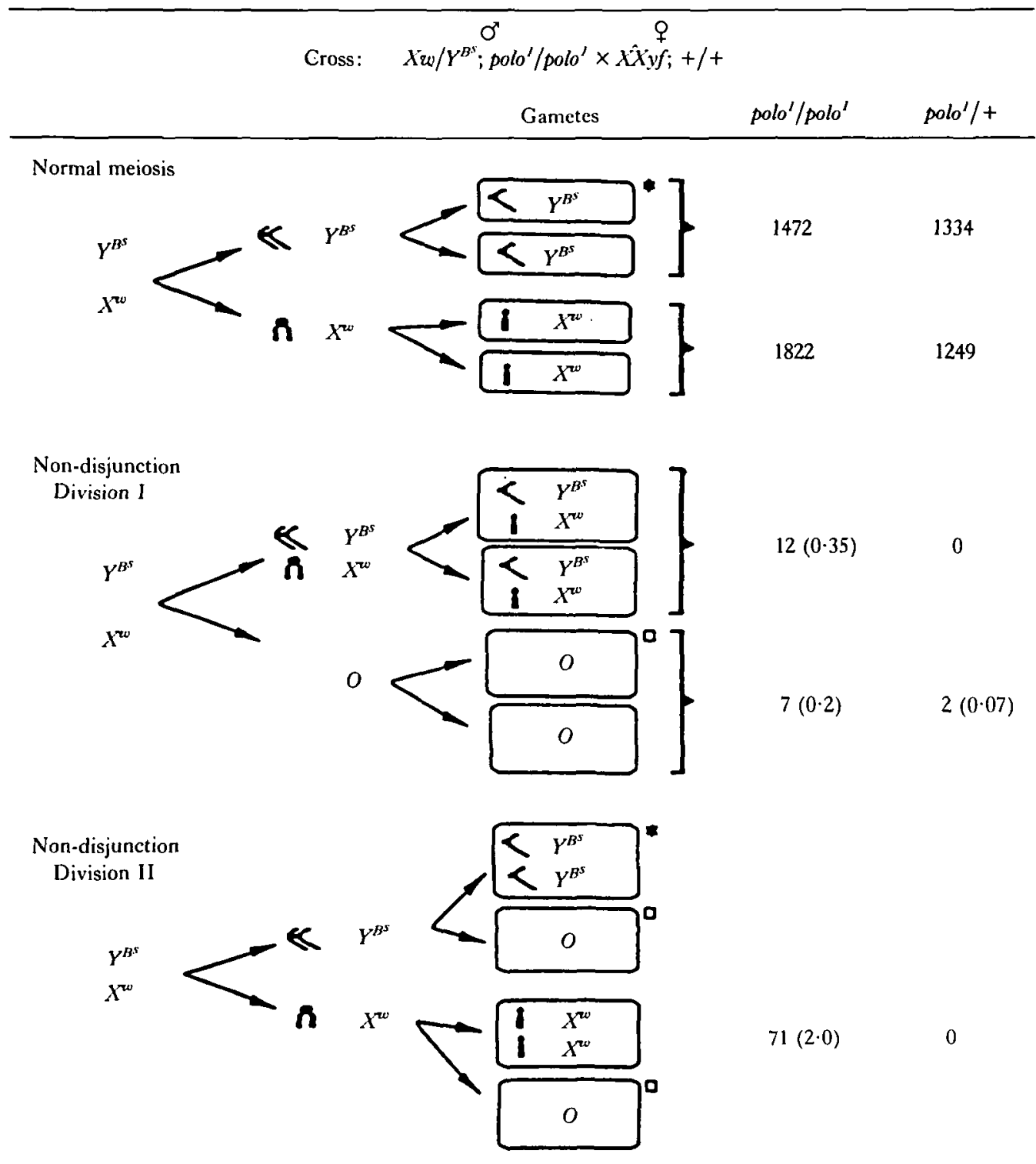

- These gametes were counted together.

$\square$ nullo gametes cannot be differentiated and therefore are counted together. The numbers in parenthesis refer to percentages of total gametes recovered.

gametes (which ought to be recoverable) could then be explained if the sex chromosome and second chromosomes were segregating into separate gametes. These possibilities are currently under investigation.

\section{The maternal effect}

The small proportion of homozygous polo ${ }^{1}$ females that survive to become adults lay a large number of eggs when mated with either Oregon R or polo $1 /$ polo $^{1}$ males. However, none of these 'polo' embryos hatch. Fig. 4 shows representative polo embryos that have been allowed to develop for increasing amounts of time before fixing and staining with the fluorescent dye,
Hoechst 33258. DNA replication and nuclear multiplication seem to proceed in a disorganized way, resulting in polyploid nuclei. Some polyploid nuclei are already evident after about $30 \mathrm{~min}$ of development (Fig. 4A), and these increase in number between 1 and $2.5 \mathrm{~h}$ (Fig. 4B,C). The appearance of these mutant embryos contrasts with the wild-type embryo shown in Fig. $4 \mathrm{~F}$. This embryo, which is from roughly mid-way through this period (cell cycle 11), has regularly spaced nuclei of equal sizes. The wild-type embryo is syncytial until the 14 th nuclear division cycle, at which point the individual nuclei become cellularized. The nuclei of polo embryos, on the other hand, never cellularize, and the nuclei continue both to increase in number and 
Table 2. Genetic non-disjunction test for the sex and second chromosomes in polo $^{1} /$ polo $^{1}$ males

Male
Cross: $\lambda w / Y B^{s} ; 2 / 2 ;$ polo $1 /$ polo $^{1} \times X / X ; C(2) E N, b w$

\begin{tabular}{|c|c|c|}
\hline \multirow{2}{*}{$\begin{array}{l}\text { Sex chromosomes in } \\
\text { male gametes }\end{array}$} & \multicolumn{2}{|c|}{ Second chromosomes in male gametes } \\
\hline & 0 & $2 / 2$ \\
\hline$X^{* v}$ & $37(14)$ & $74(29)$ \\
\hline$Y B^{s}, Y B^{S} Y B^{s *}$ & $45(17)$ & $62(24)$ \\
\hline$A^{w v} Y B^{s}$ & - & $1(0 \cdot 3)$ \\
\hline$X^{70} X^{70}$ & NR & NR \\
\hline$O$ & $2(0 \cdot 7)$ & $39(15)$ \\
\hline
\end{tabular}

The genotypes of gametes are shown that result from a crass with polo $1 /$ polo $^{1}$ fathers. An analogous experiment with polo $1 /$ polo $^{+}$ fathers gave no progeny as expected (see the text). The numbers in parenthesis refer to the percentages of total gametes recovered. NR refers to gametes that were not expected to be recovered because the progeny have low viability. Gametes of this class marked with an asterisk were scored together.

to attain high levels of polyploidy up to $6-9 \mathrm{~h}$ (Fig. 4D,E). Thereafter, there seems to be general deterioration of the embryo and breakdown of the nuclei.

A more detailed examination of polo embryos in the early stages of their development shows that their nuclei appear to be at comparable stages of the division cycle. Thus the majority of nuclei in any given embryo appear to have chromatin that is either undergoing condensation, or that appears in anaphase, or that is decondensing into telophase and subsequent interphase (data not shown). Furthermore, the nuclear envelope, as revealed by staining with an anti-lamin antibody, also seems to undergo synchronous changes in parallel with the chromatin condensation cycle as is observed of the nuclear lamin in wild-type embryos (Fuchs et al. 1983). This suggests that, although nuclei are polyploid and look very abnormal, they still undergo many of the normal cyclical aspects of mitosis.

The condensed chromatin in these early polo embryos was, however, highly disorganized and appeared as if it were not segregating correctly upon mitotic spindles. We therefore chose to examine the mitotic apparatus more closely by immuno-staining using antibodies that recognize tubulin or the centrosome. In the wild-type embryo, microtubules are nucleated by centrosomes to give asters during interphase. These split and migrate to the positions of the spindle poles during prometaphase. In metaphase, the microtubules of the spindle pole become attached to the condensed chromosomes at their kinetochores. The centrosome remains a 'focal point' for the spindle pole, and some astral microtubules still persist (see Karr \& Alberts, 1986). An example of a field of prometaphase nuclei from a wild-type embryo is shown in Fig. 5G-I.
These nuclei are regularly spaced and contain condensed chromatin $(\mathrm{G})$; the mitotic spindle has formed (H) and distinct centrosome staining can be scen with Bx63 antibody at the spindle poles (I). Examples of the microtubules of polo embryos are shown in panels $B$ and $E$. In the first field, condensed chromatin $(A)$ is associated with spindle-like microtubular structures that seem to be undergoing anaphase (B). Many of these spindle structures share one or both of their poles. Furthermore, many have very broad poles in contrast to wild-type spindles in which the polar microtubules converge on the dot-like centrosome. Surprisingly, there is no evidence of centrosomes associated with these highly branched structures and Bx63 staining is not apparent (C). At no stage of development were we able to observe asters of microtubules in the polo embryos in association with interphase-like nuclei, as in wild-type embryos (data not shown). Furthermore, there was no evidence of free asters in the cytoplasm as is observed with gnu embryos (Freeman et al. 1986) and with several other mitotic mutants (Leibowitz \& Glover, unpublished results). In some embryos, however, we observed complex microtubular structures mainly associated with condensed chromatin, but resembling complex astral structures more than spindles (E). In this field, the Bx63 antibody illuminates scattered particulate matter in the cytoplasm ( $F)$. The absence of centrosome staining by Bx63 caused us to wonder whether the polo' mutation might eliminate or cause a structural change to the antigen recognized by this antibody. We therefore carried out Western blotting experiments to look for this antigen within polo embryos. We were, however, unable to detect any difference in either molecular weight or abundance of the Bx63 antigen between wildtype or polo embryos (data not shown). We suspect therefore that centrosomal components are not organized into discrete structures in early polo embryos. When we examine later polo embryos using the Bx63 antibody, however, we are able to detect several welldefined punctate bodies that are associated with polyploid nuclei containing decondensed chromatin (Fig. 6), but never appear to be associated with microtubules.

\section{Discussion}

We have described a locus, polo, that is essential for mitosis in Drosophila, and have examined the phenotypes of polo mutants in both larval and embryonic stages of development. A larva homozygous for polo ${ }^{1}$ can survive through embryogenesis because its heterozygous mother has provided sufficient polo $^{+}$gene product for embryonic development. Most of the ensuing larval development involves cell growth, with the endoreduplication of DNA in the cells of many 

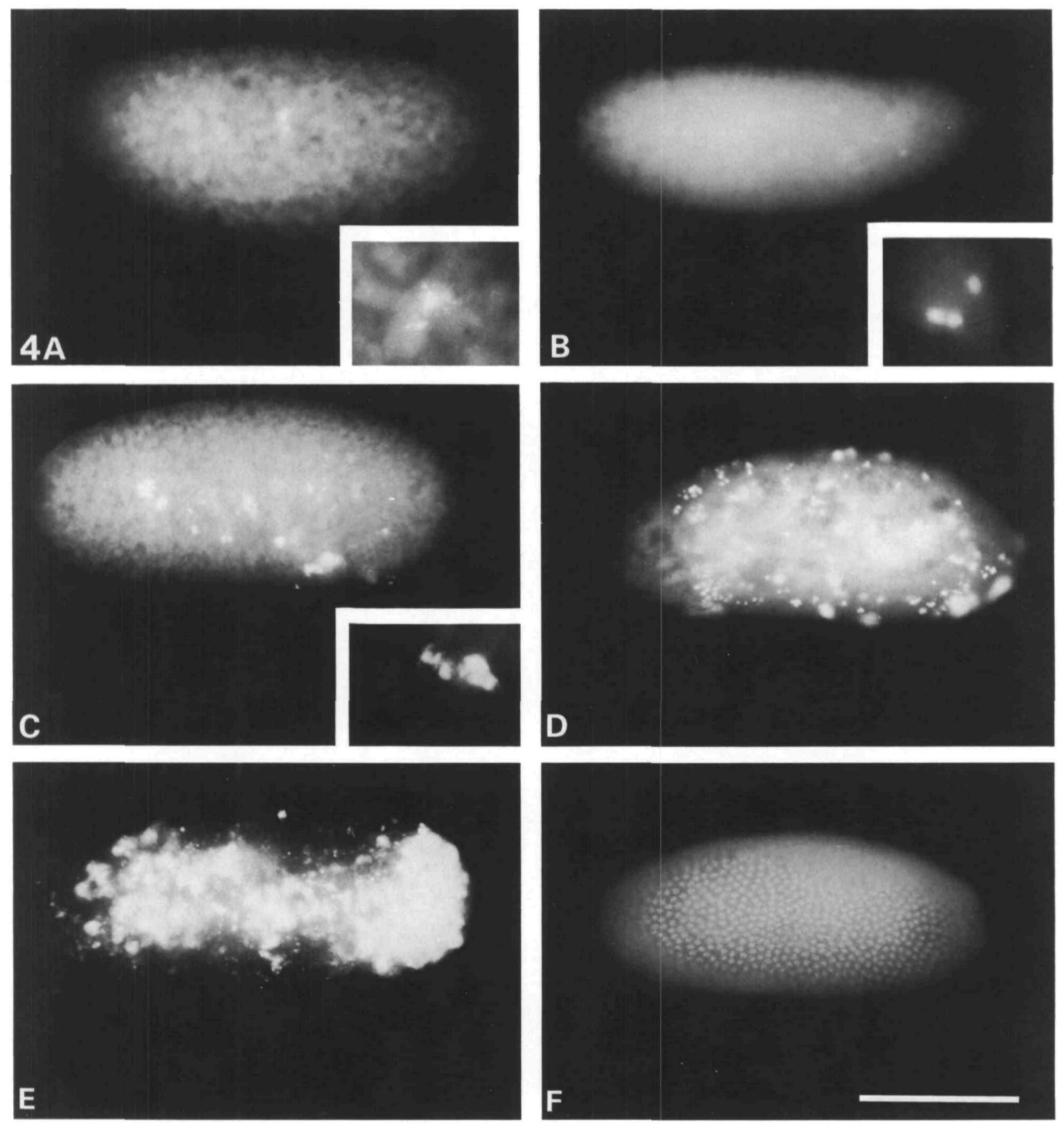

Fig. 4. The development of polo embryos. Embryos were collected for $30 \mathrm{~min}(\mathrm{~A})$ and allowed to develop at $25^{\circ} \mathrm{C}$ for a further $30 \mathrm{~min}(\mathrm{~B}), 120 \mathrm{~min}$ (C), $5 \mathrm{~h}$ (D) and $9 \mathrm{~h}$ (E). They were then fixed and stained with Hoechst. A representative embryo is shown in each panel. A wild-type embryo at cycle 11 is shown in $\mathrm{F}$. Bar, $30 \mu \mathrm{m}$. The insets in A-C show detail of the chromatin structure and are at a fourfold higher magnification.

tissues in the absence of mitosis. The diploid imaginal cells of the larva are not themselves necessary for its survival. In homozygous polo $^{2}$ larvae, these cells are eventually blocked in their mitotic division, and so become unable to proliferate to form adult structures and the organism dies in the larval stage of development. In this respect, polo falls into a class of mitotic mutants that have a late larval lethal phenotype, first recognized in the analysis of repair-defective mutants (Baker et al. 1982). In the case of the polo' allele, however, a few homozygous animals survive to adulthood and the females can produce embryos, which begin to develop but show abnormal mitoses at a very early stage of embryogenesis. The embryo develops of the order of a hundred or so polyploid nuclei and never undergoes cellularization. 
Hoechst
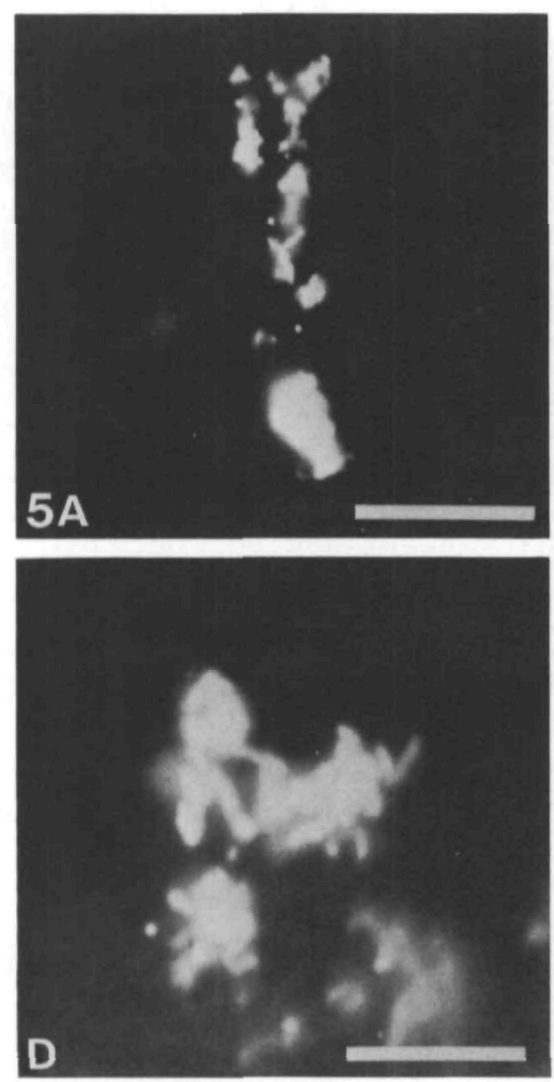

\section{B}

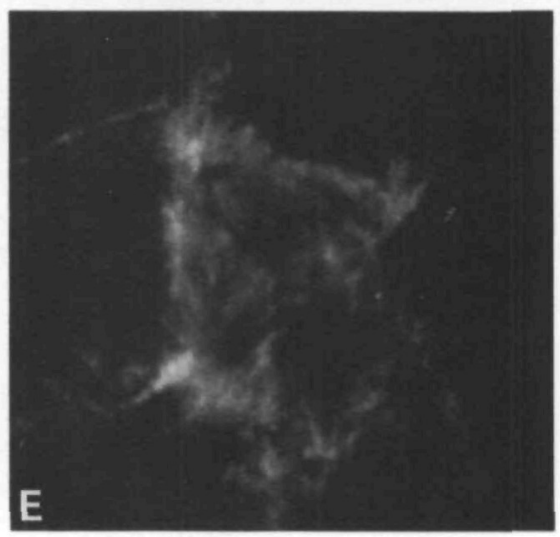

Bx63
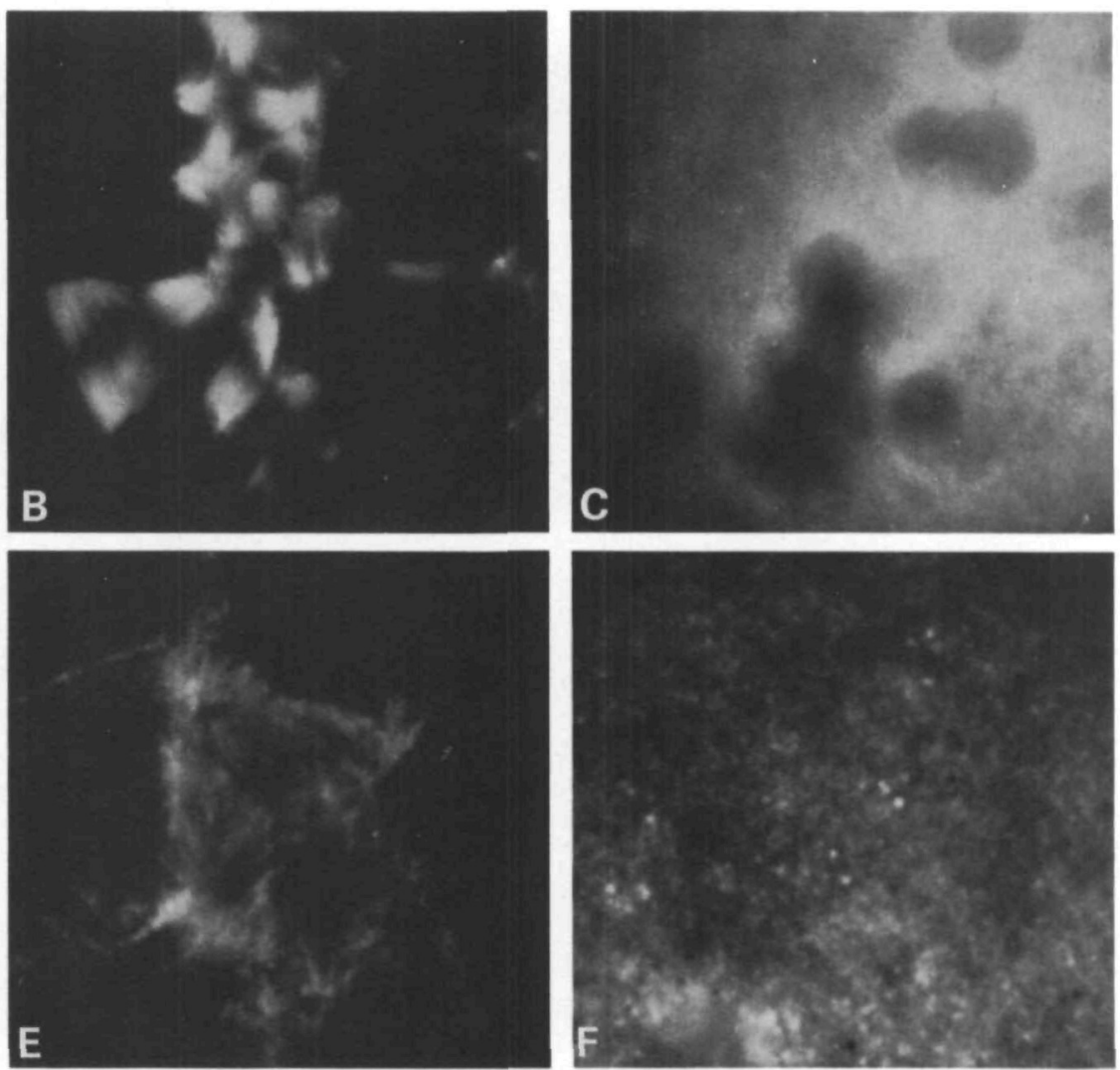
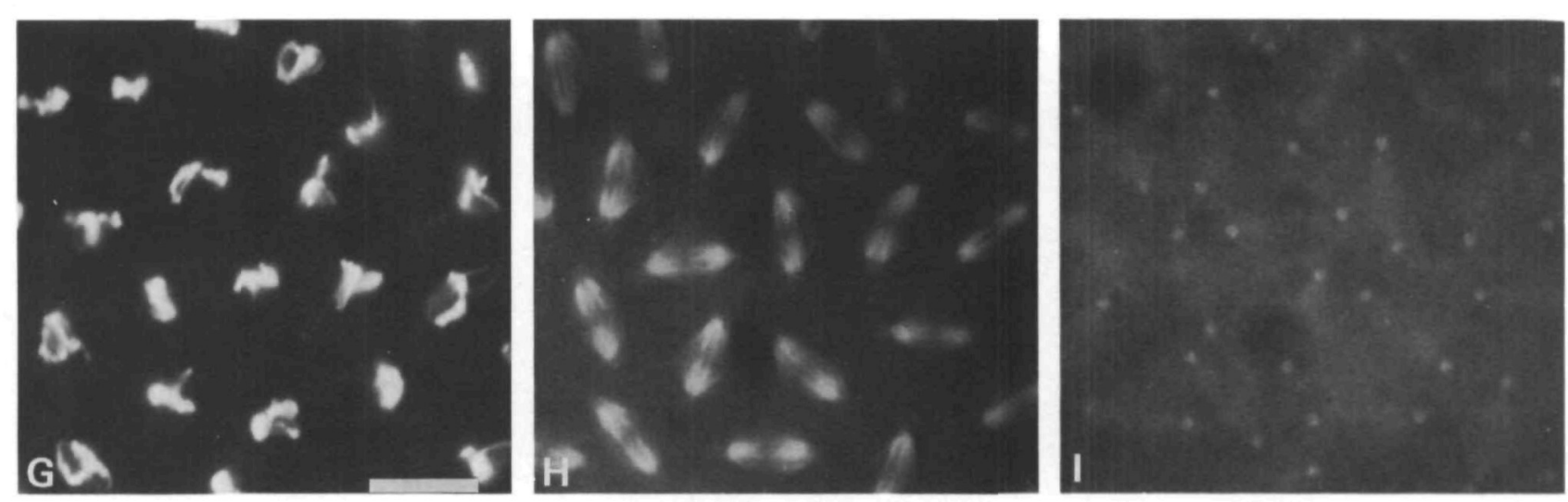

Fig. 5. Double immunostaining of polo embryos with antibodies against tubulin and centrosomes. Embryos were incubated sequentially with Bx63 antibody (mouse anti-centrosome), rhodamine-conjugated anti-mouse IgG, Y $\mathrm{Y}^{\prime} \mathrm{LI} / 2$ (rat anti-tubulin), fuorescein-conjugated anti-rat $\operatorname{IgG}$, and Hoechst (see Materials and methods). A-F. Stainings of two different polo $1 /$ polo embryos; G-1, staining of a wild-type control. Bars: A, $10 \mu \mathrm{m}, \mathrm{D}, 5 \mu \mathrm{m}, \mathrm{G}, 10 \mu \mathrm{m}$.

In very early embryos, the nuclei are evidently undergoing cyclical changes to their nuclear membranes reflected by the patterns of lamin staining, and these appear to be synchronized with chromosome condensation, metaphase and anaphase. Whilst these cyclical aspects of mitosis are retained, at least for the early nuclear cycles, the morphology of the mitotic spindles is grossly abnormal. Virtually all the spindles are branched and multipolar and, rather than having tightly focused poles, many are broad and barrel- shaped. This could be explained by a change in the structure of the centrosome. Barrel-shaped spindles are found in plant cells, for example, which differ from most animal cells in lacking centrioles and a tightly localized spindle microtubule-nucleating centre. Another indication of defective centrosomes is the absence of asters associated with interphase nuclei in polo embryos, unlike wild-type embryos. In several other mitotic mutants, we have observed free centrosomes that are dissociated from nuclei or condensed 

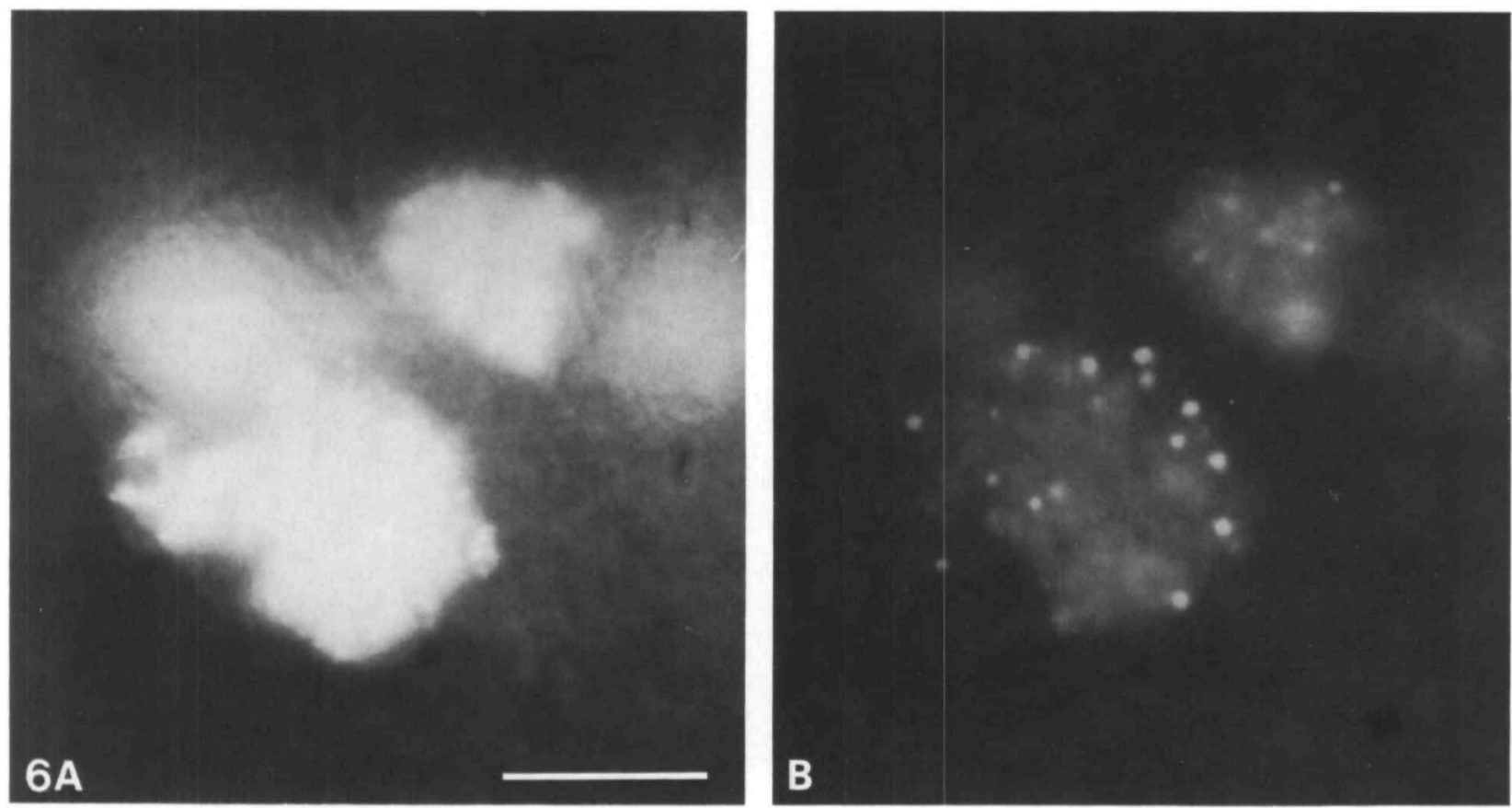

Fig. 6. Nuclear staining of a late polo embryo with Bx63 antibody. Polyploid nuclei from a late polo embryo from an 8-h collection, stained with Hoechst (A) and with the anti-centrosome antibody $B \times 63$ (B). Bar, $5 \mu \mathrm{m}$.

chromatin. The most extreme example is $g n u$, in which there is no mitosis and yet the centrosomes replicate and nucleate asters of microtubules (Freeman et al. 1986). We can also see free asters dissociated from abnormal spindles that are found in other mutants, for example, lodestar, aurora and thule (Leibowitz \& Glover, unpublished data). These mutant embryos contrast with polo embryos, in which we are unable to detect any free cytoplasmic asters.

Immunostaining of POLO embryos with the monoclonal antibody, Bx63, against an antigen associated with the centrosome indicates that POLO centrosomes are abnormal. In the wild-type embryo, the Bx63 antibody stains a large dot either at the centre of asters or at the poles of the spindle (Frasch, 1985; Frasch et al. 1986; Freeman et al. 1986). When we stain polo embryos with $\mathrm{Bx} 63$, we see either no distinct staining, or dispersed, very fine punctate staining with no obvious association with the spindle poles. Later in development, as the polo nuclei become obviously polyploid, the antigen appears to coalesce in large punctate aggregates apparently associated with the nuclear membranes of interphase nuclei. The Bx63 antigen is present throughout these stages, however, in levels comparable to wild-type embryos, as indicated by Western blotting experiments. Together these observations suggest that the polo mutation might affect a protein required for the correct structure and function of the centrosome, although such a protein need not necessarily be a centrosome component perse.
The abundance of tubulin within the larval brain hinders the immunocytological examination of spindles in neuroblasts, and so our observations have been made only upon stained chromosome preparations. The phenotype that we observe in larval neuroblasts could be explained by a lesion affecting the centrosome. The most common mitotic aberrations that we observe in these cells are polyploid circular mitotic figures, and anaphase figures in which the chromosomes progressing towards one of the poles are randomly oriented. Circular mitotic figures have also been observed in the mutant merry-go-round ( $m g r$ ) (Gonzalez, 1986; Gonzalez et al. 1987), and the mutant lodestar (Leibowitz \& Glover, unpublished data), both of which fully complement polo. They could arise if one of the spindle poles was defective with the consequent distortion of the mitotic plate as all the chromosomes are pulled to the single remaining pole. This would also explain the polyploidy that we frequently observe in these cells and in mitotic figures of this type. The abnormal anaphase structures could be a weaker manifestation of this phenotype in which one spindle pole behaves normally, and the other is only weakly affected. In the wild type, the spindle elongates during anaphase and the poles move further apart, suggesting that motive forces acting on the centrosome play an important role. One can speculate that the random orientation of the chromosomes around one of the spindle poles could be a consequence of inadequate transfer of motive force through a defective centrosome. We are unable to detect circular mitotic figures in brains from polo homozygotes treated with colchicine. Gonzalez et al. 
(1987) reported a similar finding for $m g r$, and furthermore have shown that circular figures do not form when the mutation abnormal spindle (Ripoll et al. 1985 ) is present together with $m g r$. It seems therefore that a functional spindle is required to generate circular figures. Other examples of circular mitotic figures in association with functional monopolar spindles have been observed in primary cultures of newt epithelial cells (Bajer, 1982); in a mutant line of Syrian hamster ovary cells (Wang et al. 1983); and following the inactivation of centrosome replication with $\beta$-mercaptoethanol (Mazia, 1960).

polo differs from $m g r$ in male meiosis by showing a variety of abnormal multipolar spindles in live cells during spermatogenesis. Furthermore, the spermatids have a wide range of nuclear diameters, indicating a heterogeneous DNA content resulting from chromosome non-disjunction. Mitochondrial derivatives also segregate on the meiotic spindle and then aggregate as Nebenkern in the spermatids. The Nebenkern are also heterogeneous in size in the mutant, in contrast to the wild type. This indicates that the abnormal segregation is a property associated with the spindle rather than the kinetochore of the condensed chromosomes. Our genetic analysis of non-disjunction during male meiosis suggests that the mutation affects mainly the second division. This is not to say that there is no effect on the first division, and indeed gametes indicative of this are recovered at a frequency higher than in wild-type controls. Aberrant segregation in the first meiotic division, or even in the pre-meiotic divisions of spermatogenesis, may lead to a 'meiotic catastrophe' in which the resulting gametes could be lost. The first meiotic division differs from mitotic divisions in that the centromeres do not split and instead the homologous chromosomes are segregated to the spindle poles. Therefore, a separate set of functions is required during this meiotic division. The second equational division is, on the other hand, comparable to mitosis and requires division of the centromeres. Over 40 mutants have been described that affect meiosis in Drosophila (see Baker \& Hall, 1976; Lindsley \& Sandler, 1977, for reviews). The majority of these affect the first meiotic division, different sets of genes being used for this division in males and females, reflecting the restriction to females of the processes of recombination, distributive disjunction and the formation of synaptonemal complexes. Baker et al. (1978), however, have shown that of these mutants affecting the first division, six recombination-defective loci and four loci required for correct meiotic segregation have an effect on mitotic chromosome behaviour. Mutants that preferentially affect the second meiotic division are comparatively rare. Baker \& Hall (1976) suggested that this might be because these genes would be expected to play a crucial role in mitosis and so would not have been recovered in the mutant selection schemes employed to search for meiotic mutants. Only two other loci, eq and meiS332, behave similarly to polo in this respect. The gametes produced by mei-S332 males are indicative of a relative frequency of non-disjunction in the first and second meiotic divisions comparable to that we observe with polo males (Goldstein, 1980). meiS322, however, tends to produce an excess of nullo-gametes like most other mutations that affect meiosis in Drosophila and this contrasts with our observations on polo. The low recovery of nullo-gametes in polo males could reflect their selective loss in the tests that we have carried out. It appears, however, that simultaneous non-disjunction is occurring at a high frequency. In a test designed specifically to look for the simultaneous non-disjunction of the sex and second chromosomes, we have been able to recover a high proportion of nullo- $X^{\circ}$ gametes that were in addition diplo-2, but there were very few nullo- 2 ;nullo- $X$ gametes. This raises the possibility, currently under further investigation, that disjunction of non-homologous chromosomes is occurring in polo males. This incorrect segregation of chromatids could well reflect a defect in a component of the spindle apparatus. It could be that for correct chromatid segregation to occur there have to be equal forces provided by both spindle poles. This would go some way to explaining the meiotic phenotype of polo.

Although on balance our observations point towards the polo defect affecting the function and/or organization of the centrosome, it is still prudent to exercise caution in the interpretation of mutant phenotypes. This is especially true of cyclical processes such as mitosis, which involve many interacting components. A given mutation affecting one step in the cycle could subsequently have pleiotropic consequences. Our discovery of a dysgenically induced polo allele paves the way towards cloning the gene and thereby studying the gene product. We are currently in the process of identifying the P-element that is specifically associated with the lethal polo ${ }^{2}$ mutation. This P-element-tagged DNA segment will then be cloned from a library of recombinant DNA from the mutant, permitting a full molecular analysis of the protein encoded by polo.

We are most grateful to the Cancer Research Campaign for supporting this work and for a Career Development Award to D.M.G. We thank Christianne Nusslein-Volhard for allowing us to screen her collection of third chromosome maternal effect mutants from which we isolated the polo ${ }^{1}$ allele. We are extremely grateful to Roger Karess and Alan Cheshire for their efforts in establishing a collection of dysgenic third chromosome mutants in this laboratory from which we isloated polo ${ }^{2}$. We thank Cayetano Gonzalez for teaching us about male meiosis, for improving our brain-squashing techniques, and together with Pedro Ripoll for communicating results prior to publication. Many colleagues struggled 
through the draft manuscript and we thank them for their helpful criticisms.

\section{References}

BAJer, A. (1982). Functional autonomy of monopolar spindles and evidence for oscillatory movement in mitosis. 7. Cell Biol. 93, 33-48.

Baker, B. S., Carpenter, A. T. C. \& Ripoll, P. (1978). The utilisation during mitotic cell division of loci controlling meiotic recombination and disjunction in Drosophila melanogaster. Genetics 90, 531-578.

BAKER, B. S. \& HALL, J. C. (1976). Meiotic mutants: genetic control of meiotic recombination and chromosome segregation. In The Genetics and Biology of Drosophila (ed. M. Ashburner \& E. Novitski), vol. 1a, chap. 9. London: Academic Press.

BAKER, B. S. \& SMITH, D. A. (1979). The effects of mutagen-sensitive mutants of Drosophila melanogaster in nonmutagenised cells. Genetics 110, 647-670.

BaKer, B. S., Smith, D. A. \& GatTi, M. (1982). Region specific effects on chromosome integrity of mutations at essential loci in Drosophila melanogaster. Proc. natn. Acad. Sci. U.S.A. 79, 1205-1209.

Beach, D., Durkacz, B. \& Nurse, P. (1982). Functionally homologous cell cycle control genes in budding and fission yeast. Nature, Lond. 300, 706-709.

Dequin, R., Saumweber, H. \& Sedat, J. W. (1984). Proteins shifting from the cytoplasm into the nuclei during early embryogenesis of Drosophila melanogaster. Devl Biol. 104, 37-48.

FRASCH, M. (1985). Charakerisierung chromatinassoziierter Kernproteine von Drosophila melanogaster mit Hilfe monoclonaler Antikoerper. Doctoral dissertation, Eberhard-Karls-Universität, Tübingen, FDR.

Frasch, M., Glover, D. M. \& Saumweber, H. (1986). Nuclear antigens follow different pathways into daughter nuclei during mitosis in early Drosophila embryos. $\mathcal{F}$. Cell Sci. 82, 155-172.

Freeman, M. \& Glover, D. M. (1987). The gnu mutation causes inappropriate DNA synthesis in unfertilised and fertilised eggs. Genes and Development (in press).

Freeman, M., Nusslein-Volhard, C. \& Glover, D. M. (1986). The dissociation of nuclear and centrosomal division in gmu, a mutation causing giant nuclei in Drosophila. Cell 46, 457-468.

Fuchs, J.-P., Giloh, H., Kuo, C-H., Saumweber, H. \& SEDAT, J. (1983). Nuclear structure: determination of the fate of the nuclear envelope in Drosophila during mitosis using monoclonal antibodies $\mathcal{Y}$. Cell. Sci. 64, 331-349.
GoldSTEIN, L. (1980). Mechanisms of chromosome orientation revealed by two meiotic mutants in Drosophila. Chromosoma 78, 79-111.

Gonzalez, C. (1986). Analisis genetico de la segregation cromosomica en Drosphila melanogaster. Doctoral thesis, Universiad Autonoma de Madrid.

Gonzalez, C., Casal, J. \& Ripoll, P. (1987). Functional monopolar spindles caused by mutation in mgr, a cell division gene of Drosophila melanogaster. F. Cell Sci. 89, 39-47.

Hartwell, L. H., Culotti, J., Pringle, J. R. \& Reid, B. J. (1974). Genetic control of the cell division cycle in yeast. Science 183, 46-51.

KarR, T. L. \& Alberts, B. M. (1986). Organisation of the cytoskeleton in early Drosophila embryos. Y. Cell Biol. 102, 1494-1509.

Kilmartin, J. V., Wright, B. \& Milstein, C. (1982). Rat monoclonal antitubulin antibodies derived by using a new nonsecreting rat cell line. 7. Cell Biol. 93, 576-582.

Lindsley, D. L. \& Grell, E. H. (1968). Genetic variations of Drosophila. Carnegie Institute of Washington publication no. 627.

Lindsley, D. L. \& Sandler, L. (1977). The genetic analysis of meiosis in female Drosophila melanogaster. Phil. Trans. Roy. Soc Lond. B, 277, 295-312.

MAZIA, D. (1960). In L'action antimitotique et caryoclasique de substances chemiques (ed. J. Turchini \& P. Sentein), p. 167. Paris: Colloque no. 88 CNRS.

NurSE, P. (1985). Cell cycle control genes in yeast. Trends Genet. 1, 51-55.

Pringle, J. \& Hartwell, L. (1981). The Saccharomyces cerevisiae cell cycle. In The Molecular Biology of the Yeast Saccharomyces (ed. S. Strathern, E. Jones \& J. Broach), pp. 97-142. New York: Cold Spring Harbor Laboratory Press.

Ripoll, P., Pimpinelli, S., Valdivia, M. M. \& Avilı, J. (1985). A cell division mutant of Drosophila with a functionally abnormal spindle. Cell 41, 907-912.

Sandler, L., Lindsley, D. L., Nicoletti, B. \& Trippa, G. (1968). Mutants affecting meiosis in natural populations of Drosophila melanogaster. Genetics 60, 528-558.

Smith, D. A., Baker, B. S. \& Gatti, M. (1985). Mutations in genes encoding essential mitotic functions in Drosophila melanogaster. Genetics 110, 647-670.

WANG, R. J., Wissinger, W., King, E. J. \& WANG, G. (1983). Studies on cell division in mammalian cells. VII. A temperature sensitive cell line abnormal in centriole separation and chromosome movement. Y. Cell Biol 96, 301-306.

ZalokAR, M. \& ERK, I. (1976). Division and migration of nuclei during early embryogenesis of Drosophila melanogaster. Y. Microsc. Biol. Cell. 25, 97-106.

(Received 13 August 1987 -Accepted 18 September 1987) 